TRANSACTIONS OF THE

AMERICAN MATHEMATICAL SOCIETY

Volume 365, Number 6, June 2013, Pages 3355-3372

S 0002-9947(2013)05859-X

Article electronically published on January 4, 2013

\title{
ON EPSILON FACTORS ATTACHED TO SUPERCUSPIDAL REPRESENTATIONS OF UNRAMIFIED U $(2,1)$
}

\author{
MICHITAKA MIYAUCHI
}

Abstract. Let $G$ be the unramified unitary group in three variables defined over a $p$-adic field $F$ with $p \neq 2$. Gelbart, Piatetski-Shapiro and Baruch attached zeta integrals of Rankin-Selberg type to irreducible generic representations of $G$. In this paper, we formulate a conjecture on $L$ - and $\varepsilon$-factors defined through zeta integrals in terms of newforms for $G$, which is an analogue of the result by Casselman and Deligne for GL(2). We prove our conjecture for the generic supercuspidal representations of $G$.

\section{INTRODUCTION}

Local newforms play an important role in the theory of automorphic forms. Casselman and Deligne established a theory of newforms for $p$-adic GL(2), which can be stated as follows. Let $F$ be a non-archimedean local field of characteristic zero with the ring of integers $\mathfrak{o}_{F}$ and its maximal ideal $\mathfrak{p}_{F}$. Let $\psi_{F}$ be a nontrivial additive character of $F$ with conductor $\mathfrak{o}_{F}$. The local counterpart of a level subgroup of $\mathrm{GL}_{2}(F)$ is defined by

$$
\Gamma_{0}\left(\mathfrak{p}_{F}^{n}\right)=\left(\begin{array}{cc}
\mathfrak{o}_{F} & \mathfrak{o}_{F} \\
\mathfrak{p}_{F}^{n} & 1+\mathfrak{p}_{F}^{n}
\end{array}\right)^{\times},
$$

for $n \geq 0$. For each irreducible admissible representation $(\pi, V)$ of $\mathrm{GL}_{2}(F)$, we define the subspace

$$
V(n)=\left\{v \in V \mid \pi(k) v=v, k \in \Gamma_{0}\left(\mathfrak{p}_{F}^{n}\right)\right\}
$$

of $V$. Then the following theorem holds.

Theorem $1.1([2])$. Let $(\pi, V)$ be an irreducible generic representation of $\mathrm{GL}_{2}(F)$.

(i) There exists a non-negative integer $n$ such that $V(n) \neq\{0\}$.

(ii) Put $c(\pi)=\min \{n \mid V(n) \neq\{0\}\}$. Then the space $V(c(\pi))$ is one-dimensional.

(iii) The $\varepsilon$-factor $\varepsilon\left(s, \pi, \psi_{F}\right)$ of $\pi$ is a constant multiple of $q_{F}^{-c(\pi) s}$, where $q_{F}$ is the cardinality of the residue field of $F$.

We call the integer $c(\pi)$ the conductor of $\pi$ and $V(c(\pi))$ the space of newforms for $\pi$. Another important property of newforms is that the zeta integral of a newform expresses the $L$-factor of a representation.

Theorem $1.2([3])$. Let $\pi$ be an irreducible generic representation of $\mathrm{GL}_{2}(F)$ and $W$ a newform in the Whittaker model of $\pi$. Then the corresponding JacquetLanglands's zeta integral $Z(s, W)$ attains the $L$-factor of $\pi$.

Received by the editors August 1, 2011 and, in revised form, March 23, 2012 and April 16, 2012 .

2010 Mathematics Subject Classification. Primary 22E50, 22E35.

Key words and phrases. p-adic group, local newform, $\varepsilon$-factor.

(C) 2013 American Mathematical Society Reverts to public domain 28 years from publication 
Similar results were obtained by Jacquet, Piatetski-Shapiro and Shalika [6] for $\mathrm{GL}_{n}(F)$. Recently, Roberts and Schmidt [10] established a theory of newforms for irreducible representations of $\mathrm{GSp}_{4}(F)$ whose central characters are trivial. Our main concern is to construct a newform theory for unramified unitary group $\mathrm{U}(2,1)$.

The aim of this paper is twofold. The first one is to formulate an analogue of Theorem 1.2 for Gelbart and Piatetski-Shapiro's zeta integrals for unramified $\mathrm{U}(2,1)$. Gelbart and Piatetski-Shapiro 4 initiated a theory of Rankin-Selberg integrals for $\mathrm{U}(2,1)$, and Baruch 1 developed it. For a given irreducible generic representation of $\mathrm{U}(2,1)$, they attached a zeta integral involving a Whittaker function and a Schwartz function on $F^{2}$. The $L$-factor attached to an irreducible generic representation of $\mathrm{U}(2,1)$ is defined as the greatest common divisor of the zeta integrals. For unramified $\mathrm{U}(2,1)$, the author 8 introduced a family of open compact subgroups and defined newforms for generic representations $\pi$. We conjecture that the $L$-factor of $\pi$ is represented by the zeta integral when the Whittaker function is associated to the newform and the Schwartz function is the characteristic function of a certain lattice in $F^{2}$ (Conjecture 4.1). In addition, our conjecture indicates the relation between $\varepsilon$-factors and conductors (Theorem 4.3). We note that there is a related work by Koseki and Oda [7] for the archimedean situation.

The second aim of this paper is to show that our conjecture holds for the generic supercuspidal representations. The zeta integral involving the newform and the characteristic function of a certain lattice in $F^{2}$ is decomposed into a product of $L_{E}(s, \mathbf{1})$ and a more simple zeta integral of the newform, which is close to that for GL(2) (Proposition 3.5). Here $E$ is the unramified quadratic extension over $F$ and $L_{E}(s, \mathbf{1})$ stands for the $L$-factor of the trivial representation of $\mathrm{GL}_{1}(E)$. To compute the latter zeta integral, we follow the method by Roberts and Schmidt for GSp(4). In [10], they utilized Hecke operators acting on the space of newforms to obtain a key formula of the values of the Whittaker function associated to the newform at diagonal matrices in terms of Hecke eigenvalues. The zeta integral of the newform is determined by this formula. In their theory, the assumption on the central character is crucial. We apply their method to representations of unramified $\mathrm{U}(2,1)$ whose conductors differ from those of their central characters to obtain an explicit formula of zeta integrals of newforms in terms of Hecke eigenvalues (Proposition 5.12). All the supercuspidal representations satisfy this assumption on the central characters, so our conjecture holds for them.

We summarize the contents of this paper. In section 2, we fix the basic notation for representations of the unramified unitary group in three variables and recall the notion of its newforms. In section 3, we recall from [1] the theory of Rankin-Selberg integral for unramified $\mathrm{U}(2,1)$ and give some computation relating to newforms. In section 4, we give Conjecture 4.1, which says that zeta integrals of newforms attain the $L$-factors of representations. In section [5, we introduce the Hecke operator and the level lowering operator, and give their explicit description. Using these operators, we get a formula of zeta integrals of newforms in terms of Hecke eigenvalues. In section 6. we show that our conjecture is true for all the generic supercuspidal representations of unramified $\mathrm{U}(2,1)$.

Unfortunately, our conjecture is still open for non-supercuspidal representations. For such representations, conductors may be equal to those of central characters, so our technique in section 5 does not work. We will develop a theory of Hecke operators for such representations, and show the validity of the conjecture in our 
ongoing work. It is also an important problem to compare $L$ - and $\varepsilon$-factors defined through zeta integrals with those of $L$-parameters.

\section{Preliminaries}

In subsection 2.1, we fix our notation for unramified $\mathrm{U}(2,1)$, which is used in this paper. In subsection 2.2, we recall from [8] the definition and basic properties of newforms for unramified $\mathrm{U}(2,1)$.

2.1. Notation. Let $F$ be a non-archimedean local field of characteristic zero, $\mathfrak{o}_{F}$ its ring of integers, and $\mathfrak{p}_{F}=\varpi_{F} \mathfrak{o}_{F}$ the maximal ideal in $\mathfrak{o}_{F}$. We write $q=q_{F}$ for the cardinality of $\mathfrak{o}_{F} / \mathfrak{p}_{F}$. Let $|\cdot|_{F}$ denote the absolute value of $F$ normalized so that $\left|\varpi_{F}\right|_{F}=q_{F}^{-1}$. We use the analogous notation for any non-archimedean local field. Throughout this paper, we assume that the residual characteristic of $F$ is odd.

Let $E=F[\sqrt{\epsilon}]$ be the unramified quadratic extension over $F$, where $\epsilon$ is a nonsquare unit in $\mathfrak{o}_{F}$. We know that $q_{E}=q^{2}$ and $\varpi_{F}$ is a uniformizer of $E$. So we abbreviate $\varpi=\varpi_{F}$. We set $G=\left\{g \in \mathrm{GL}_{3}(E) \mid{ }^{t} \bar{g} J g=J\right\}$, where ${ }^{-}$is the non-trivial element in $\operatorname{Gal}(E / F)$ and

$$
J=\left(\begin{array}{lll}
0 & 0 & 1 \\
0 & 1 & 0 \\
1 & 0 & 0
\end{array}\right)
$$

Then $G$ is a realization of the $F$-points of unramified $\mathrm{U}(2,1)$ defined over $F$. We denote by $e$ the identity element of $G$.

Let $B$ be the Borel subgroup of $G$ consisting of the upper triangular elements in $G$ with Levi subgroup $T$ of diagonal matrices in $G$ and unipotent radical $U$. We write $\hat{U}$ for the opposite of $U$ :

$$
\begin{aligned}
& U=\left\{u(x, y)=\left(\begin{array}{ccc}
1 & x & y \\
0 & 1 & -\bar{x} \\
0 & 0 & 1
\end{array}\right) \mid x, y \in E, y+\bar{y}+x \bar{x}=0\right\}, \\
& \hat{U}=\left\{\hat{u}(x, y)=\left(\begin{array}{ccc}
1 & 0 & 0 \\
x & 1 & 0 \\
y & -\bar{x} & 1
\end{array}\right) \mid x, y \in E, y+\bar{y}+x \bar{x}=0\right\} .
\end{aligned}
$$

We shall identify the subgroup

$$
H=\left\{\left(\begin{array}{ccc}
a & 0 & b \\
0 & 1 & 0 \\
c & 0 & d
\end{array}\right) \in G\right\}
$$

of $G$ with $\mathrm{U}(1,1)$. We set $B_{H}=B \cap H, U_{H}=U \cap H$ and $T_{H}=T \cap H$ :

$$
T_{H}=\left\{t(a)=\left(\begin{array}{ccc}
a & 0 & 0 \\
0 & 1 & 0 \\
0 & 0 & \bar{a}^{-1}
\end{array}\right) \mid a \in E^{\times}\right\} .
$$

For $a \in E^{\times}$, we put $t(a)=\left(\begin{array}{cc}a & 0 \\ 0 & \bar{a}^{-1}\end{array}\right)$ and $d(a)=\left(\begin{array}{cc}a & 0 \\ 0 & 1\end{array}\right)$. Then every element $h$ in $H=\mathrm{U}(1,1)$ can be decomposed into

$$
h=t(b) d(\sqrt{\epsilon}) h_{1} d\left(\sqrt{\epsilon}^{-1}\right),
$$

where $b \in E^{\times}$and $h_{1} \in \mathrm{SL}_{2}(F)$. 
For a non-trivial additive character $\psi_{E}$ of $E$, we define a character $\psi_{E}$ of $U$ by

$$
\psi_{E}(u(x, y))=\psi_{E}(x) \text {, for } u(x, y) \in U .
$$

We say that a smooth representation $(\pi, V)$ of $G$ is generic if $\operatorname{Hom}_{U}\left(\pi, \psi_{E}\right) \neq\{0\}$. Suppose that $(\pi, V)$ is irreducible and generic. Then it is well-known that

$$
\operatorname{dim} \operatorname{Hom}_{U}\left(\pi, \psi_{E}\right)=1 .
$$

By Frobenius reciprocity, there exists a unique embedding of $\pi$ into $\operatorname{Ind}_{U}^{G} \psi_{E}$ up to scalar. The image $\mathcal{W}\left(\pi, \psi_{E}\right)$ of $V$ is called the Whittaker model of $\pi$. By a nonzero functional $l \in \operatorname{Hom}_{U}\left(\pi, \psi_{E}\right)$, we define the Whittaker function $W_{v} \in \mathcal{W}\left(\pi, \psi_{E}\right)$ associated to $v \in V$ by

$$
W_{v}(g)=l(\pi(g) v), g \in G .
$$

We may identify the center $Z$ of $G$ with the norm-one subgroup $E^{1}$ of $E^{\times}$. Under this identification, we set open compact subgroups of $Z$ as

$$
Z_{0}=Z, Z_{n}=Z \cap\left(1+\mathfrak{p}_{E}^{n}\right) \text {, for } n \geq 1 .
$$

For an irreducible admissible representation $\pi$ of $G$, we denote by $\omega_{\pi}$ the central character of $\pi$. We define the conductor of $\omega_{\pi}$ by

$$
n_{\pi}=\min \left\{n \geq 0\left|\omega_{\pi}\right|_{Z_{n}}=1\right\} .
$$

2.2. Newforms. For a non-negative integer $n$, we define an open compact subgroup $K_{n}$ of $G$ by

$$
K_{n}=\left(\begin{array}{ccc}
\mathfrak{o}_{E} & \mathfrak{o}_{E} & \mathfrak{p}_{E}^{-n} \\
\mathfrak{p}_{E}^{n} & 1+\mathfrak{p}_{E}^{n} & \mathfrak{o}_{E} \\
\mathfrak{p}_{E}^{n} & \mathfrak{p}_{E}^{n} & \mathfrak{o}_{E}
\end{array}\right) \cap G .
$$

For a smooth representation $(\pi, V)$ of $G$, we denote by $V(n)$ the space of $K_{n}$-fixed vectors in $V$, namely,

$$
V(n)=\left\{v \in V \mid \pi(k) v=v, k \in K_{n}\right\}, n \geq 0 .
$$

Theorem 2.2 ([8] Theorems 2.8, 5.6, Corollary 5.5(i)). Suppose that $(\pi, V)$ is an irreducible generic representation of $G$.

(i) There exists a non-negative integer $n$ such that $V(n) \neq\{0\}$.

(ii) Put $N_{\pi}=\min \{n \geq 0 \mid V(n) \neq\{0\}\}$. Then $\operatorname{dim} V\left(N_{\pi}\right)=1$.

(iii) If $\pi$ is supercuspidal, then we have $N_{\pi} \geq 2$ and $N_{\pi}>n_{\pi}$.

Definition 2.3 (8] Definition 2.6). Let $(\pi, V)$ be an irreducible generic representation of $G$. We call $N_{\pi}$ the conductor of $\pi$ and $V\left(N_{\pi}\right)$ the space of newforms for $\pi$.

We recall some properties of Whittaker functions associated to newforms. Let $(\pi, V)$ be an irreducible generic representation of $G$. For each $v \in V$, we can regard $\left.W_{v}\right|_{T_{H}}$ as a locally constant function on $E^{\times}$. Along the lines of the Kirillov theory for GL(2), we see that there exists an integer $n$ such that supp $\left.W_{v}\right|_{T_{H}}$ is contained in $\mathfrak{p}_{E}^{-n}$. Moreover, if $v$ is an element in $\langle\pi(u) w-w \mid u \in U, w \in V\rangle$, then $\left.W_{v}\right|_{T_{H}}$ is a compactly supported function on $E^{\times}$.

Proposition 2.4 ( 8 . Corollary 4.6, Theorem 4.12). Suppose that $\psi_{E}$ has conductor $\mathfrak{o}_{E}$. Let $\pi$ be an irreducible generic representation of $G$ and let $v$ be a newform for $\pi$.

(i) The function $\left.W_{v}\right|_{T_{H}}$ is $\mathfrak{o}_{E}^{\times}$-invariant and its support is contained in $\mathfrak{o}_{E}$.

(ii) Suppose that $N_{\pi} \geq 2$ and $N_{\pi}>n_{\pi}$. Then $W_{v}(e)=0$ if and only if $v=0$. 


\section{RANKin-SELBERG INTEGRAL}

In this section, we recall from [1] the theory of the Rankin-Selberg integral for unramified $\mathrm{U}(2,1)$ by Gelbart, Piatetski-Shapiro and Baruch, and give some computation relating to newforms. For an irreducible generic representation $\pi$ of $G$, its zeta integral has the form $Z(s, W, \Phi)$, where $W$ is a Whittaker function for $\pi$ and $\Phi$ is a Schwartz function on $F^{2}$. In subsection 3.1, we see that $Z(s, W, \Phi)$ can be decomposed into a product of a more simple zeta integral and the $L$-factor of the trivial representation of $E^{\times}$when $W$ is associated to a newform for $\pi$ and $\Phi$ is the characteristic function of a certain lattice in $F^{2}$. The $L$-factor attached to $\pi$ is defined as the greatest common divisor of the zeta integrals. In subsection 3.2 . we quote the result by Ishikawa on the shape of $L$-factors of supercuspidal representations. In subsection 3.3 , we recall their functional equation, and relate it with contragradient representations. In subsection 3.4 we show that $\varepsilon$-factors are monomial.

3.1. Zeta integrals. Let $\mathcal{C}_{c}^{\infty}\left(F^{2}\right)$ be the space of locally constant, compactly supported functions on $F^{2}$. For $\Phi \in \mathcal{C}_{c}^{\infty}\left(F^{2}\right)$ and $g \in \mathrm{GL}_{2}(F)$, we define a function $g \Phi$ in $\mathcal{C}_{c}^{\infty}\left(F^{2}\right)$ by

$$
(g \Phi)(x, y)=\Phi((x, y) g),(x, y) \in F^{2} .
$$

We normalize the Haar measure on $F^{\times}$so that the volume of $\mathfrak{o}_{F}^{\times}$is one. For $\Phi \in \mathcal{C}_{c}^{\infty}\left(F^{2}\right)$ and $g \in \mathrm{GL}_{2}(F)$, we define a function $z(s, g, \Phi)$ on $\mathbf{C}$ by

$$
z(s, g, \Phi)=\int_{F^{\times}}(g \Phi)(0, r)|r|_{E}^{s} d^{\times} r, s \in \mathbf{C} .
$$

For any subset $S$ of $F^{2}$, we denote by $\operatorname{ch}_{S}$ the characteristic function of $S$. We set $\Phi_{n}=\operatorname{ch}_{\mathfrak{p}_{F}^{n} \oplus \mathfrak{o}_{F}}$, for each integer $n$. We define the $L$-factor $L_{E}(s, \chi)$ of a quasicharacter $\chi$ of $E^{\times}$as usual:

$$
L_{E}(s, \chi)=\left\{\begin{array}{cl}
\frac{1}{1-\chi(\varpi) q^{-2 s}}, & \text { if } \chi \text { is unramified } \\
1, & \text { if } \chi \text { is ramified. }
\end{array}\right.
$$

We denote by 1 the trivial character of $E^{\times}$.

Lemma 3.1. For any $k \in\left(\begin{array}{cc}\mathfrak{o}_{F} & \mathfrak{p}_{F}^{-n} \\ \mathfrak{p}_{F}^{n} & \mathfrak{o}_{F}\end{array}\right)^{\times}$, we have $z\left(s, k, \Phi_{n}\right)=L_{E}(s, \mathbf{1})$.

Proof. Since $k \in\left(\begin{array}{cc}\mathfrak{o}_{F} & \mathfrak{p}_{F}^{-n} \\ \mathfrak{p}_{F}^{n} & \mathfrak{o}_{F}\end{array}\right)^{\times}$fixes the lattice $\mathfrak{p}_{F}^{n} \oplus \mathfrak{o}_{F}$, we have $k \Phi_{n}=\Phi_{n}$. Thus we get

$$
z\left(s, k, \Phi_{n}\right)=\int_{F^{\times}} \Phi_{n}(0, r)|r|_{E}^{s} d^{\times} r=\int_{\mathfrak{o}_{F} \cap F^{\times}}|r|_{F}^{2 s} d^{\times} r=L_{E}(s, \mathbf{1}),
$$

as required.

For $h \in H$ and $\Phi \in \mathcal{C}_{c}^{\infty}\left(F^{2}\right)$, we set

$$
f(s, h, \Phi)=|b|_{E}^{s} z\left(s, h_{1}, \Phi\right), s \in \mathbf{C},
$$

where $b \in E^{\times}$and $h_{1} \in \mathrm{SL}_{2}(F)$ are as in (2.1). By [1] Lemma 2.5, the definition of $f(s, h, \Phi)$ is independent of the choice of $b \in E^{\times}$and $h_{1} \in \mathrm{SL}_{2}(F)$. Set $K_{n, H}=$ 
$K_{n} \cap H$. We may identify $K_{n, H}$ with $\left(\begin{array}{cc}\mathfrak{o}_{E} & \mathfrak{p}_{E}^{-n} \\ \mathfrak{p}_{E}^{n} & \mathfrak{o}_{E}\end{array}\right) \cap \mathrm{U}(1,1)$. Because $K_{n, H}$ is a good maximal compact subgroup of $H$, we have an Iwasawa decomposition $H=U_{H} T_{H} K_{n, H}$. We note that

$$
f(s, t(a) k, \Phi)=|a|_{E}^{s} f(s, k, \Phi), a \in E^{\times}, k \in K_{n, H} .
$$

Lemma 3.3. For any $k \in K_{n, H}$, we have $f\left(s, k, \Phi_{n}\right)=L_{E}(s, \mathbf{1})$.

Proof. We can decompose $k \in K_{n, H}$ as $k=t(b) d(\sqrt{\epsilon}) k_{1} d\left(\sqrt{\epsilon}^{-1}\right)$, where $b \in \mathfrak{o}_{E}^{\times}$and $k_{1} \in\left(\begin{array}{cc}\mathfrak{o}_{F} & \mathfrak{p}_{F}^{-n} \\ \mathfrak{p}_{F}^{n} & \mathfrak{o}_{F}\end{array}\right)^{\times} \cap \mathrm{SL}_{2}(F)$. So we obtain $f(s, k, \Phi)=|b|_{E}^{s} z\left(s, k_{1}, \Phi\right)=L_{E}(s, \mathbf{1})$ by Lemma 3.1 .

Let $\pi$ be an irreducible generic representation of $G$. For $W \in \mathcal{W}\left(\pi, \psi_{E}\right)$ and $\Phi \in \mathcal{C}_{c}^{\infty}\left(F^{2}\right)$, we define the zeta integral

$$
Z(s, W, \Phi)=\int_{U_{H} \backslash H} W(h) f(s, h, \Phi) d h .
$$

By [1] Proposition 3.4, the integral $Z(s, W, \Phi)$ absolutely converges to a function in $\mathbf{C}\left(q^{-2 s}\right)$ when $\operatorname{Re}(s)$ is sufficiently large. We normalize the Haar measures on $E^{\times}$and $K_{n, H}$ so that the volumes of $\mathfrak{o}_{E}^{\times}$and of $K_{n, H}$ are one respectively. By the Iwasawa decomposition $H=U_{H} T_{H} K_{n, H}$ and the isomorphism $E^{\times} \simeq T_{H} ; a \mapsto t(a)$, we obtain

$$
Z(s, W, \Phi)=\int_{K_{n, H}} \int_{E^{\times}} W(t(a) k) f(s, t(a) k, \Phi)|a|_{E}^{-1} d^{\times} a d k .
$$

We define another zeta integral of $W$ in $\mathcal{W}\left(\pi, \psi_{E}\right)$ by

$$
Z(s, W)=\int_{E^{\times}} W(t(a))|a|_{E}^{s-1} d^{\times} a .
$$

By the proof of [1] Proposition 3.4, $Z(s, W)$ also converges absolutely to a function in $\mathbf{C}\left(q^{-2 s}\right)$ if $\operatorname{Re}(s)$ is large enough.

Proposition 3.5. Suppose that $W \in \mathcal{W}\left(\pi, \psi_{E}\right)$ is fixed by $K_{n, H}$. Then we have

$$
Z\left(s, W, \Phi_{n}\right)=Z(s, W) L_{E}(s, \mathbf{1}) .
$$

Proof. If $W \in \mathcal{W}\left(\pi, \psi_{E}\right)$ is fixed by $K_{n, H}$, then we get

$$
\begin{aligned}
Z\left(s, W, \Phi_{n}\right) & =\int_{K_{n, H}} \int_{E \times} W(t(a))|a|_{E}^{s-1} f\left(s, k, \Phi_{n}\right) d^{\times} a d k \\
& =\int_{E^{\times}} W(t(a))|a|_{E}^{s-1} d^{\times} a \cdot \int_{K_{n, H}} f\left(s, k, \Phi_{n}\right) d k
\end{aligned}
$$

by (3.2) and (3.4). Now the assertion follows from Lemma 3.3 and the definition of $Z(s, W)$. 
3.2. $L$-factors. Let $I_{\pi}$ be the subspace of $\mathbf{C}\left(q^{-2 s}\right)$ spanned by $Z(s, W, \Phi)$ where $\Phi \in \mathcal{C}_{c}^{\infty}\left(F^{2}\right), W \in \mathcal{W}\left(\pi, \psi_{E}\right)$ and $\psi_{E}$ runs over the non-trivial additive characters of $E$. As remarked in [1] p. 331, $I_{\pi}$ is a fractional ideal of $\mathbf{C}\left(q^{-2 s}\right)$. So we can find a polynomial $P(X) \in \mathbf{C}[X]$ such that $P(0)=1$ and $1 / P\left(q^{-2 s}\right)$ generates $I_{\pi}$. We define the $L$-factor $L(s, \pi)$ of $\pi$ by

$$
L(s, \pi)=\frac{1}{P\left(q^{-2 s}\right)} .
$$

The following proposition is due to Ishikawa, but the statement is slightly modified.

Proposition 3.6 ([5] Theorem 4(4)). Let $\pi$ be an irreducible generic supercuspidal representation of $G$. Then $L(s, \pi)$ equals to 1 or $L_{E}(s, \mathbf{1})$.

Proof. It is enough to show that the function $Z(s, W, \Phi) / L_{E}(s, \mathbf{1})$ belongs to $\mathbf{C}\left[q^{-2 s}, q^{2 s}\right]$ for all $W \in \mathcal{W}\left(\pi, \psi_{E}\right), \Phi \in \mathcal{C}_{c}^{\infty}\left(F^{2}\right)$ and non-trivial additive characters $\psi_{E}$ of $E$. Since $W(h)$ and $f(s, h, \Phi)$ are right smooth with respect to $h \in H$, the integral $Z(s, W, \Phi)$ is a linear combination of functions of the form $Z\left(s, W^{\prime}\right) f\left(s, \mathbf{1}, \Phi^{\prime}\right)$, where $W^{\prime} \in \mathcal{W}\left(\pi, \psi_{E}\right)$ and $\Phi^{\prime} \in \mathcal{C}_{c}^{\infty}\left(F^{2}\right)$. It follows from [8] Propositions 4.1(ii) and 4.7 that $\left.W^{\prime}\right|_{T_{H}}$ is a compactly supported function on $T_{H} \simeq E^{\times}$. Note that in [8], we assume that $\psi_{E}$ has conductor $\mathfrak{o}_{E}$, but Proposition 4.7 holds for all $\psi_{E}$. This implies that $Z\left(s, W^{\prime}\right)$ lies in $\mathbf{C}\left[q^{-2 s}, q^{2 s}\right]$. Due to the theory of zeta integrals for $\mathrm{GL}(1)$, we see that $f\left(s, \mathbf{1}, \Phi^{\prime}\right) / L_{E}(s, \mathbf{1})$ belongs to $\mathbf{C}\left[q^{-2 s}, q^{2 s}\right]$. This completes the proof.

3.3. The functional equation. Let $\psi_{F}$ be a non-trivial additive character of $F$ with conductor $\mathfrak{p}_{F}^{c\left(\psi_{F}\right)}$. We choose the Haar measure on $F^{2}$ normalized so that the volume of $\mathfrak{o}_{F} \oplus \mathfrak{o}_{F}$ is $q^{c\left(\psi_{F}\right)}$. For each $\Phi \in \mathcal{C}_{c}^{\infty}\left(F^{2}\right)$, we define the Fourier transform $\hat{\Phi}$ by

$$
\hat{\Phi}(x, y)=\int_{F^{2}} \Phi(u, v) \psi_{F}(y u-x v) d u d v
$$

One can check that $\hat{\hat{\Phi}}=\Phi$ for all $\Phi \in \mathcal{C}_{c}^{\infty}\left(F^{2}\right)$.

Lemma 3.7. Suppose that the conductor of $\psi_{F}$ is $\mathfrak{o}_{F}$. Then we have

$$
z\left(1-s, k, \hat{\Phi}_{n}\right)=q^{-2 n(s-1 / 2)} L_{E}(1-s, \mathbf{1}),
$$

for any $k \in\left(\begin{array}{cc}\mathfrak{o}_{F} & \mathfrak{p}_{F}^{-n} \\ \mathfrak{p}_{F}^{n} & \mathfrak{o}_{F}\end{array}\right)^{\times}$

Proof. It is easy to observe that $\hat{\Phi}_{n}=q^{-n} \operatorname{ch}_{\mathfrak{o}_{F} \oplus \mathfrak{p}_{F}^{-n}}$. Since $\hat{\Phi}_{n}$ is fixed by $k$, we get

$$
\begin{aligned}
z\left(1-s, k, \hat{\Phi}_{n}\right) & =q^{-n} \int_{F^{\times}} \operatorname{ch}_{\mathfrak{o}_{F} \oplus \mathfrak{p}_{F}^{-n}}(0, r)|r|_{E}^{1-s} d^{\times} r \\
& =q^{-n} \int_{\mathfrak{p}_{F}^{-n} \cap F^{\times}}|r|_{E}^{1-s} d^{\times} r \\
& =q^{-2 n(s-1 / 2)} L_{E}(1-s, \mathbf{1}),
\end{aligned}
$$

as required.

Corollary 3.8. If the conductor of $\psi_{F}$ is $\mathfrak{o}_{F}$, then we have $f\left(1-s, k, \hat{\Phi}_{n}\right)=$ $q^{-2 n(s-1 / 2)} L_{E}(1-s, \mathbf{1})$, for $k \in K_{n, H}$. 
Proof. Exactly the same as the proof of Lemma 3.3.

Proposition 3.9. Suppose that $\psi_{F}$ has conductor $\mathfrak{o}_{F}$. If a Whittaker function $W$ in $\mathcal{W}\left(\pi, \psi_{E}\right)$ is fixed by $K_{n, H}$, then we have

$$
Z\left(1-s, W, \hat{\Phi}_{n}\right)=q^{-2 n(s-1 / 2)} Z\left(1-s, W, \Phi_{n}\right) .
$$

Proof. By the proof of Proposition 3.5, we get

$$
Z\left(1-s, W, \hat{\Phi}_{n}\right)=Z(1-s, W) \cdot \int_{K_{n, H}} f\left(1-s, k, \hat{\Phi}_{n}\right) d k
$$

Due to Corollary 3.8, one has $Z\left(1-s, W, \hat{\Phi}_{n}\right)=q^{-2 n(s-1 / 2)} Z(1-s, W) L_{E}(1-s, \mathbf{1})$. So we get $Z\left(1-s, W, \hat{\Phi}_{n}\right)=q^{-2 n(s-1 / 2)} Z\left(1-s, W, \Phi_{n}\right)$ by Proposition 3.5. that

By [1] Corollary 4.8, there exists a rational function $\gamma\left(s, \pi, \psi_{F}, \psi_{E}\right)$ in $q^{-2 s}$ such

$$
\gamma\left(s, \pi, \psi_{F}, \psi_{E}\right) Z(s, W, \Phi)=Z(1-s, W, \hat{\Phi}) .
$$

Note that in the right hand side we do not take the contragradient of $W$. To extract the $\varepsilon$-factor of $\pi$ from our integrals, the above relation is the right form of the local functional equation. We explain this in more detail in the remaining of this subsection.

For an irreducible admissible representation $(\pi, V)$ of $G$, we denote by $\tilde{\pi}$ its contragradient representation, and by $\bar{\pi}$ the representation of $G$ on $V$ defined by

$$
\bar{\pi}(g)=\pi(\bar{g}), g \in G .
$$

Lemma 3.11. Let $\pi$ be an irreducible admissible representation of $G$. Then $\tilde{\pi}$ is isomorphic to $\bar{\pi}$.

Proof. We define a Hermitian form $h$ on $E^{3}$ by

$$
h(v, w)={ }^{t} \bar{v} J w, v, w \in E^{3} .
$$

Then $G$ is just the group of isometries of $\left(E^{3}, h\right)$. Let $\delta$ be the element in $\operatorname{Aut}_{F} E^{3}$ defined by $\delta v=\bar{v}$ for $v \in E^{3}$. Then it follows from [9] p. 91 that $\widetilde{\pi}$ is isomorphic to $\pi^{\delta}$ where $\pi^{\delta}(g)=\pi\left(\delta g \delta^{-1}\right)$, for $g \in G$. Since $\pi^{\delta}(g)=\bar{\pi}(g)$, for $g \in G$, the lemma follows.

We further assume $\pi$ is generic. For $W \in \mathcal{W}\left(\pi, \psi_{E}\right)$, we set

$$
\bar{W}(g)=W(\bar{g}), g \in G .
$$

By Lemma 3.11, we see that $\bar{W}$ lies in $\mathcal{W}\left(\bar{\pi}, \bar{\psi}_{E}\right)=\mathcal{W}\left(\widetilde{\pi}, \bar{\psi}_{E}\right)$, where $\bar{\psi}_{E}$ is the character of $U$ given by $\bar{\psi}_{E}(u)=\psi_{E}(\bar{u}), u \in U$. We define another Fourier transformation on $\mathcal{C}_{c}^{\infty}\left(F^{2}\right)$ as follows:

$$
\Phi^{*}(x, y)=\int_{F^{2}} \Phi(u, v) \psi_{F}(y u+x v) d u d v, \Phi \in \mathcal{C}_{c}^{\infty}\left(F^{2}\right) .
$$

We shall rewrite the right hand side of (3.10) as a zeta integral of $\tilde{\pi}$.

Lemma 3.12. For $W \in \mathcal{W}\left(\pi, \psi_{E}\right)$ and $\Phi \in \mathcal{C}_{c}^{\infty}\left(F^{2}\right)$, we have

$$
Z(s, W, \hat{\Phi})=Z\left(s, \bar{W}, \Phi^{*}\right) \text {. }
$$


Proof. We claim that $d(-1) \Phi^{*}=\hat{\Phi}$. In fact, we have

$$
\begin{aligned}
\left(d(-1) \Phi^{*}\right)(x, y) & =\Phi^{*}((x, y) d(-1))=\Phi^{*}(-x, y) \\
& =\int_{F^{2}} \Phi(u, v) \psi_{F}(y u-x v) d u d v=\hat{\Phi}(x, y),
\end{aligned}
$$

for $(x, y) \in F^{2}$. Thus, for $g \in \mathrm{GL}_{2}(F)$, we obtain

$$
\begin{aligned}
z\left(s, d(-1) g d(-1), \Phi^{*}\right) & =\int_{F^{\times}}\left(d(-1) g d(-1) \Phi^{*}\right)(0, r)|r|_{E}^{s} d^{\times} r \\
& =\int_{F^{\times}}(g \hat{\Phi})((0, r) d(-1))|r|_{E}^{s} d^{\times} r \\
& =\int_{F^{\times}}(g \hat{\Phi})(0, r)|r|_{E}^{s} d^{\times} r=z(s, g, \hat{\Phi}) .
\end{aligned}
$$

Next, we shall show that $f\left(s, \bar{h}, \Phi^{*}\right)=f(s, h, \hat{\Phi})$ for all $h \in H$. Suppose that $h \in H$ is written as $h=t(b) d(\sqrt{\epsilon}) h_{1} d\left(\sqrt{\epsilon}^{-1}\right)$, where $b \in E^{\times}$and $h_{1} \in \operatorname{SL}_{2}(F)$. Then we get

$$
\begin{aligned}
\bar{h} & =t(\bar{b}) d(\overline{\sqrt{\epsilon}}) \bar{h}_{1} d\left(\overline{\sqrt{\epsilon}}^{-1}\right)=t(\bar{b}) d(-\sqrt{\epsilon}) h_{1} d\left(-\sqrt{\epsilon}^{-1}\right) \\
& =t(\bar{b}) d(\sqrt{\epsilon}) d(-1) h_{1} d(-1) d\left(\sqrt{\epsilon}^{-1}\right) .
\end{aligned}
$$

Since $d(-1) h_{1} d(-1)$ lies in $\mathrm{SL}_{2}(F)$ and the section $f$ is independent of the choice of $b$ and $h_{1}$, we have $f\left(s, \bar{h}, \Phi^{*}\right)=|\bar{b}|_{E}^{s} z\left(s, d(-1) h_{1} d(-1), \Phi^{*}\right)=|b|_{E}^{s} z\left(s, h_{1}, \hat{\Phi}\right)=$ $f(s, h, \hat{\Phi})$.

Haar measure $d h$ on $U_{H} \backslash H$ satisfies $d h=d \bar{h}$ since $U_{H}$ and $K_{n, H}$ are stable under the action of $\operatorname{Gal}(E / F)$. Therefore we get

$$
\begin{aligned}
Z\left(s, \bar{W}, \Phi^{*}\right) & =\int_{U_{H} \backslash H} \bar{W}(h) f\left(s, h, \Phi^{*}\right) d h=\int_{U_{H} \backslash H} W(\bar{h}) f\left(s, h, \Phi^{*}\right) d h \\
& =\int_{U_{H} \backslash H} W(h) f\left(s, \bar{h}, \Phi^{*}\right) d h=\int_{U_{H} \backslash H} W(h) f(s, h, \hat{\Phi}) d h \\
& =Z(s, W, \hat{\Phi}) .
\end{aligned}
$$

This is the asserted equation.

Lemma 3.12 tells that the $L$-factor of $\pi$ coincides with that of $\tilde{\pi}$.

Proposition 3.13. For any irreducible generic representation $\pi$ of $G$, we have $L(s, \pi)=L(s, \tilde{\pi})$.

Proof. It follows from Lemma 3.12 that the space $I_{\pi}$ coincides with $I_{\widetilde{\pi}}$. Now the assertion is obvious.

3.4. $\varepsilon$-factors. The $\varepsilon$-factor $\varepsilon\left(s, \pi, \psi_{F}, \psi_{E}\right)$ of an irreducible generic representation $\pi$ of $G$ is defined by

$$
\varepsilon\left(s, \pi, \psi_{F}, \psi_{E}\right)=\gamma\left(s, \pi, \psi_{F}, \psi_{E}\right) \frac{L(s, \pi)}{L(1-s, \tilde{\pi})} .
$$

Due to Proposition 3.13, we have

$$
\varepsilon\left(s, \pi, \psi_{F}, \psi_{E}\right)=\gamma\left(s, \pi, \psi_{F}, \psi_{E}\right) \frac{L(s, \pi)}{L(1-s, \pi)} .
$$


Proposition 3.15. The $\varepsilon$-factor $\varepsilon\left(s, \pi, \psi_{F}, \psi_{E}\right)$ is a monomial in $\mathbf{C}\left[q^{-2 s}, q^{2 s}\right]$ of the form

with some $n \in \mathbf{Z}$.

$$
\varepsilon\left(s, \pi, \psi_{F}, \psi_{E}\right)= \pm q^{-2 n(s-1 / 2)},
$$

Proof. By definition, there exist $\Phi_{i} \in \mathcal{C}_{c}^{\infty}\left(F^{2}\right)$, additive characters $\psi_{E, i}$ of $E$ and $W_{i} \in \mathcal{W}\left(\pi, \psi_{E, i}\right)(1 \leq i \leq k)$ such that

$$
\sum_{i=1}^{k} \frac{Z\left(s, W_{i}, \Phi_{i}\right)}{L(s, \pi)}=1 .
$$

By the proof of [1] Lemma 4.9, there exists $W_{i}^{\prime} \in \mathcal{W}\left(\pi, \psi_{E}\right)$ such that $Z\left(s, W_{i}, \Phi_{i}\right)=$ $q^{-2 s m_{i}} Z\left(s, W_{i}^{\prime}, \Phi_{i}\right)$, for some $m_{i} \in \mathbf{Z}$. From (3.16), we have the following expression of the $\varepsilon$-factor:

$$
\begin{aligned}
\varepsilon\left(s, \pi, \psi_{F}, \psi_{E}\right) & =\varepsilon\left(s, \pi, \psi_{F}, \psi_{E}\right) \sum_{i=1}^{k} \frac{q^{-2 s m_{i}} Z\left(s, W_{i}^{\prime}, \Phi_{i}\right)}{L(s, \pi)} \\
& =\sum_{i=1}^{k} \frac{q^{-2 s m_{i}} Z\left(1-s, W_{i}^{\prime}, \hat{\Phi}_{i}\right)}{L(1-s, \pi)} .
\end{aligned}
$$

The second equality is a consequence of (3.10) and (3.14). This implies that $\varepsilon\left(s, \pi, \psi_{F}, \psi_{E}\right)$ is a polynomial in $q^{-2 s}$ and $q^{2 s}$.

By the above expression of the $\varepsilon$-factor, we get

$$
\begin{aligned}
\varepsilon\left(s, \pi, \psi_{F}, \psi_{E}\right) \varepsilon\left(1-s, \pi, \psi_{F}, \psi_{E}\right) & =\varepsilon\left(s, \pi, \psi_{F}, \psi_{E}\right) \sum_{i=1}^{k} \frac{q^{-2(1-s) m_{i}} Z\left(s, W_{i}^{\prime}, \hat{\Phi}_{i}\right)}{L(s, \pi)} \\
& =\sum_{i=1}^{k} \frac{q^{-2(1-s) m_{i}} Z\left(1-s, W_{i}^{\prime}, \Phi_{i}\right)}{L(1-s, \pi)} \\
& =1 .
\end{aligned}
$$

In the second equality, we use the functional equation and $\hat{\hat{\Phi}}_{i}=\Phi_{i}$. The last equality is a consequence of (3.16). Now the assertion follows by standard arguments.

\section{Conjecture on NeWforms}

We give the following conjecture on zeta integrals of newforms.

Conjecture 4.1. We fix an additive character $\psi_{E}$ of $E$ with conductor $\mathfrak{o}_{E}$. Let $\pi$ be an irreducible generic representation of $G$. Then there exists a newform $v$ for $\pi$ which satisfies

$$
Z\left(s, W_{v}, \Phi_{N_{\pi}}\right)=L(s, \pi),
$$

where $N_{\pi}$ is the conductor of $\pi$ and $\Phi_{N_{\pi}}$ is the characteristic function of $\mathfrak{p}_{F}^{N_{\pi}} \oplus \mathfrak{o}_{F}$.

Here is our main theorem, which will be proved in section 6 .

Theorem 4.2. Conjecture 4.1 holds for any irreducible generic supercuspidal representations of $G$.

If Conjecture 4.1 is true, then we obtain a formula of the $\varepsilon$-factors, which says that the exponents of $q^{-2 s}$ of the $\varepsilon$-factors for generic representations agree with their conductors. 
Theorem 4.3. Let $\psi_{E}$ and $\psi_{F}$ be additive characters of $E$ and of $F$ with conductors $\mathfrak{o}_{E}$ and $\mathfrak{o}_{F}$ respectively. Assume that Conjecture 4.1 is true for an irreducible generic representation $\pi$ of $G$. Then we have

$$
\varepsilon\left(s, \pi, \psi_{F}, \psi_{E}\right)=q_{E}^{-N_{\pi}(s-1 / 2)},
$$

where $N_{\pi}$ is the conductor of $\pi$ and $q_{E}$ is the cardinality of the residue field of $E$.

Proof. By assumption, there exists a newform $v$ for $\pi$ such that $Z\left(s, W_{v}, \Phi_{N_{\pi}}\right)=$ $L(s, \pi)$. By Proposition 3.9 and the assumption, we have

$$
\begin{aligned}
Z\left(1-s, W_{v}, \hat{\Phi}_{N_{\pi}}\right) & =q^{-2 N_{\pi}(s-1 / 2)} Z\left(1-s, W_{v}, \Phi_{N_{\pi}}\right) \\
& =q^{-2 N_{\pi}(s-1 / 2)} L(1-s, \pi) .
\end{aligned}
$$

So we get

$$
\begin{aligned}
\varepsilon\left(s, \pi, \psi_{F}, \psi_{E}\right) & =\gamma\left(s, \pi, \psi_{F}, \psi_{E}\right) \frac{L(s, \pi)}{L(1-s, \pi)} \\
& =\frac{Z\left(1-s, W_{v}, \hat{\Phi}_{N_{\pi}}\right)}{Z\left(s, W_{v}, \Phi_{N_{\pi}}\right)} \frac{L(s, \pi)}{L(1-s, \pi)} \\
& =q^{-2 N_{\pi}(s-1 / 2)} .
\end{aligned}
$$

This proves the theorem.

\section{ZETA INTEGRALS OF NEWFORMS}

To show Theorem 4.2, let us compute zeta integrals of newforms for generic supercuspidal representations of $G$. It follows from Theorem 2.2(iii) that generic supercuspidal representations $(\pi, V)$ of $G$ satisfy $N_{\pi} \geq 2$ and $N_{\pi}>n_{\pi}$. Thus, we treat representations with this property in this section. In subsection [5.1, we introduce the Hecke operator $T$ on $V(n)$ and give its explicit description. If $n=N_{\pi}$, then this operator is scalar and has the Hecke eigenvalue. In subsection 5.2, we consider the level lowering operator $\delta: V(n) \rightarrow V(n-1)$ and give its explicit formula when $n \geq 2$ and $n>n_{\pi}$. Combining these results, in subsection 5.3. we get a recursion formula of the values of the Whittaker functions associated to newforms at diagonal matrices (Lemma 5.11), which gives an explicit formula of zeta integrals of newforms in terms of Hecke eigenvalues (Proposition 5.12). The results in this section are strongly inspired by those in [10] sections 6 and 7 .

5.1. Hecke operator. From now on, we fix a non-trivial additive character $\psi_{E}$ of $E$ with conductor $\mathfrak{o}_{E}$. Put

$$
\zeta=\left(\begin{array}{ccc}
\varpi & & \\
& 1 & \\
& & \varpi^{-1}
\end{array}\right) .
$$

Let $(\pi, V)$ be an irreducible generic representation of $G$ and $n$ a non-negative integer. We define the Hecke operator $T$ on $V(n)$ by

$$
T v=\frac{1}{\operatorname{vol}\left(K_{n}\right)} \int_{K_{n} \zeta K_{n}} \pi(k) v d k, v \in V(n) .
$$


Using the bijection $K_{n} / K_{n} \cap \zeta K_{n} \zeta^{-1} \simeq K_{n} \zeta K_{n} / K_{n} ; k\left(K_{n} \cap \zeta K_{n} \zeta^{-1}\right) \mapsto k \zeta K_{n}$, we can write $T v$ as

$$
T v=\sum_{k \in K_{n} / K_{n} \cap \zeta K_{n} \zeta^{-1}} \pi(k \zeta) v .
$$

We set

$$
t_{n}=\left(\begin{array}{ccc} 
& & \varpi^{-n} \\
& 1 & \\
\varpi^{n} & &
\end{array}\right) \in K_{n} .
$$

Lemma 5.2. Suppose that $n \geq 1$. Then a complete set of representatives for $K_{n} / K_{n} \cap \zeta K_{n} \zeta^{-1}$ is given by

$$
t_{n} u(y, z \sqrt{\epsilon}-y \bar{y} / 2) \text { and } u(a, b \sqrt{\epsilon}-a \bar{a} / 2),
$$

where $y, a \in \mathfrak{o}_{E} / \mathfrak{p}_{E}, z \in \mathfrak{p}_{F}^{1-n} / \mathfrak{p}_{F}^{2-n}$ and $b \in \mathfrak{p}_{F}^{-n} / \mathfrak{p}_{F}^{2-n}$.

Proof. Note that

$$
K_{n} \cap \zeta K_{n} \zeta^{-1}=\left(\begin{array}{ccc}
\mathfrak{o}_{E} & \mathfrak{p}_{E} & \mathfrak{p}_{E}^{2-n} \\
\mathfrak{p}_{E}^{n} & 1+\mathfrak{p}_{E}^{n} & \mathfrak{p}_{E} \\
\mathfrak{p}_{E}^{n} & \mathfrak{p}_{E}^{n} & \mathfrak{o}_{E}
\end{array}\right) \cap G
$$

Set

$$
K^{\prime}=\left(\begin{array}{ccc}
\mathfrak{o}_{E} & \mathfrak{o}_{E} & \mathfrak{p}_{E}^{1-n} \\
\mathfrak{p}_{E}^{n} & 1+\mathfrak{p}_{E}^{n} & \mathfrak{o}_{E} \\
\mathfrak{p}_{E}^{n} & \mathfrak{p}_{E}^{n} & \mathfrak{o}_{E}
\end{array}\right) \cap G
$$

Clearly, the following two claims assert the lemma:

(i) A complete set of representatives for $K_{n} / K^{\prime}$ is given by the $q+1$ elements $t_{n}$ and $u(0, x \sqrt{\epsilon})$, where $x \in \mathfrak{p}_{F}^{-n} / \mathfrak{p}_{F}^{1-n}$.

(ii) We can take a complete set of representatives for $K^{\prime} / K_{n} \cap \zeta K_{n} \zeta^{-1}$ as the $q^{3}$ elements $u(y, z \sqrt{\epsilon}-y \bar{y} / 2)$, where $y \in \mathfrak{o}_{E} / \mathfrak{p}_{E}, z \in \mathfrak{p}_{F}^{1-n} / \mathfrak{p}_{F}^{2-n}$.

We shall prove these claims. It is obvious that elements in (i) and (ii) belong to pairwise distinct cosets in $K_{n} / K^{\prime}$ and $K^{\prime} / K_{n} \cap \zeta K_{n} \zeta^{-1}$ respectively.

(i) We denote by $g_{i j}$ the $(i, j)$-entry of $g \in M_{3}(E)$. Let $k \in K_{n}$. If $k_{33} \in \mathfrak{p}_{E}$, then we get $t_{n} k \in K^{\prime}$, and hence $k \in t_{n} K^{\prime}$. Suppose that $k_{33} \in \mathfrak{o}_{E}^{\times}$. Since $k$ lies in $G, k$ satisfies $J^{t} \bar{k} J k=e$ (see subsection 2.1). Comparing (1,3)-entries of this equation, we have $k_{13} \bar{k}_{33}+k_{23} \bar{k}_{23}+k_{33} \bar{k}_{13}=0$. We note that $k_{13} \in \mathfrak{p}_{E}^{-n}$ and $k_{23}, k_{33} \in \mathfrak{o}_{E}$ because $k \in K_{n}$. Since $k_{13} \bar{k}_{33} \in \mathfrak{p}_{E}^{-n}$ satisfies $k_{13} \bar{k}_{33}+k_{33} \bar{k}_{13}=-k_{23} \bar{k}_{23} \in \mathfrak{o}_{F}$, one has $k_{13} \bar{k}_{33} \in \mathfrak{o}_{F} \oplus \mathfrak{p}_{F}^{-n} \sqrt{\epsilon}$. By the assumption $k_{33} \in \mathfrak{o}_{E}^{\times}$, we have $k_{33} \bar{k}_{33} \in \mathfrak{o}_{F}^{\times}$, and hence $k_{13} k_{33}^{-1}=\left(k_{33} \bar{k}_{33}\right)^{-1} k_{13} \bar{k}_{33} \in \mathfrak{o}_{F} \oplus \mathfrak{p}_{F}^{-n} \sqrt{\epsilon}$. Thus there exists $x \in \mathfrak{p}_{F}^{-n}$ such that $k_{13} k_{33}^{-1}-x \sqrt{\epsilon} \in \mathfrak{o}_{F}$. Using the assumption again, we get $k_{13}-x \sqrt{\epsilon} k_{33} \in \mathfrak{o}_{E} \subset$ $\mathfrak{p}_{E}^{1-n}$. This implies $u(0,-x \sqrt{\epsilon}) k \in K^{\prime}$, so that $k \in u(0, x \sqrt{\epsilon}) K^{\prime}$.

(ii) If $n=1$, then $K^{\prime}$ lies in the standard Iwahori subgroup of $G$. One can see that $K^{\prime}$ has an Iwahori decomposition $K^{\prime}=\left(K^{\prime} \cap \hat{U}\right)\left(K^{\prime} \cap T\right)\left(K^{\prime} \cap U\right)$. The assertion follows because $\left(K^{\prime} \cap \hat{U}\right)\left(K^{\prime} \cap T\right) \subset K_{n} \cap \zeta K_{n} \zeta^{-1}$.

Suppose that $n \geq 2$. For $k \in K^{\prime}$, we set $y=k_{22}^{-1} k_{12}$. Then $y$ lies in $\mathfrak{o}_{E}$. Since $\operatorname{det} k \in E^{1}$ and $\operatorname{det} k \equiv k_{11} k_{33}\left(\bmod \mathfrak{p}_{E}\right)$, the element $k_{33}$ must belong to $\mathfrak{o}_{E}^{\times}$. As 
in the proof of (i), we can take $z \in \mathfrak{p}_{F}^{1-n}$ such that $k_{13}-z \sqrt{\epsilon} k_{33} \in \mathfrak{o}_{E} \subset \mathfrak{p}_{E}^{2-n}$. Then one can easily check that $k$ belongs to $u(y, z \sqrt{\epsilon}-y \bar{y} / 2)\left(K_{n} \cap \zeta K_{n} \zeta^{-1}\right)$. This completes the proof.

Lemma 5.2 gives an explicit description of the Hecke operator $T$. For $v \in V(n)$, we set

$$
v^{\prime}=\sum_{y \in \mathfrak{p}_{E}^{n-1} / \mathfrak{p}_{E}^{n}} \sum_{z \in \mathfrak{p}_{F}^{n-1} / \mathfrak{p}_{F}^{n}} \pi(\hat{u}(y, z \sqrt{\epsilon}-y \bar{y} / 2)) v .
$$

Then we get the following lemma.

Lemma 5.4. Suppose that $n \geq 1$. Then we have

$$
T v=\pi\left(\zeta^{-1}\right) v^{\prime}+\sum_{a \in \mathfrak{o}_{E} / \mathfrak{p}_{E}} \sum_{b \in \mathfrak{p}_{F}^{-n} / \mathfrak{p}_{F}^{2-n}} \pi(u(a, b \sqrt{\epsilon}-a \bar{a} / 2) \zeta) v,
$$

for $v \in V(n)$.

Proof. By (5.1) and Lemma 5.2 we obtain

$$
T v=\sum_{\substack{y \in \mathfrak{o}_{E} / \mathfrak{p}_{E} \\ z \in \mathfrak{p}_{F}^{-n} / \mathfrak{p}_{F}^{2-n}}} \pi\left(t_{n} u(y, z \sqrt{\epsilon}-y \bar{y} / 2) \zeta\right) v+\sum_{\substack{a \in \mathfrak{o}_{E} / \mathfrak{p}_{E} \\ b \in \mathfrak{p}_{F}^{-n} / \mathfrak{p}_{F}^{2-n}}} \pi(u(a, b \sqrt{\epsilon}-a \bar{a} / 2) \zeta) v .
$$

Here we note that $t_{n} \in K_{n}$ so $\pi\left(t_{n}\right) v=v$, and use $\zeta t_{n}=t_{n-1}$ and

$$
t_{n-1} u(y, z \sqrt{\epsilon}-y \bar{y} / 2) t_{n-1}=\hat{u}\left(-\varpi^{n-1} \bar{y}, \varpi^{2 n-2}(z \sqrt{\epsilon}-y \bar{y} / 2)\right),
$$

then

$$
\begin{aligned}
\pi\left(t_{n} u(y, z \sqrt{\epsilon}-y \bar{y} / 2) \zeta\right) v & =\pi\left(\zeta^{-1}\right) \pi\left(\zeta t_{n} u(y, z \sqrt{\epsilon}-y \bar{y} / 2) \zeta t_{n}\right) v \\
& =\pi\left(\zeta^{-1}\right) \pi\left(\hat{u}\left(-\varpi^{n-1} \bar{y}, \varpi^{2 n-2}(z \sqrt{\epsilon}-y \bar{y} / 2)\right)\right) v
\end{aligned}
$$

Hence we get

$$
\begin{aligned}
& \sum_{\substack{y \in \mathfrak{o}_{E} / \mathfrak{p}_{E} \\
z \in \mathfrak{p}_{F}^{1-n} / \mathfrak{p}_{F}^{2-n}}} \pi\left(t_{n} u(y, z \sqrt{\epsilon}-y \bar{y} / 2) \zeta\right) v \\
= & \pi\left(\zeta^{-1}\right) \sum_{\substack{y \in \mathfrak{o}_{E} / \mathfrak{p}_{E} \\
z \in \mathfrak{p}_{F}^{1-n} / \mathfrak{p}_{F}^{2-n}}} \pi\left(\hat{u}\left(-\varpi^{n-1} \bar{y}, \varpi^{2 n-2}(z \sqrt{\epsilon}-y \bar{y} / 2)\right)\right) v \\
= & \pi\left(\zeta^{-1}\right) v^{\prime},
\end{aligned}
$$

which completes the proof.

We shall consider the case when $n=N_{\pi}$. Because $V\left(N_{\pi}\right)$ is one-dimensional, there exists $\lambda \in \mathbf{C}$ such that $T v=\lambda v$ for all $v \in V\left(N_{\pi}\right)$. We call $\lambda$ the Hecke eigenvalue of $T$. For a newform $v$ in $V\left(N_{\pi}\right)$, we put

$$
c_{i}=W_{v}\left(\zeta^{i}\right), c_{i}^{\prime}=W_{v^{\prime}}\left(\zeta^{i}\right), i \in \mathbf{Z} .
$$

Then we obtain the following lemma.

Lemma 5.6. Suppose that $N_{\pi} \geq 1$. Then we have

$$
\lambda c_{i}=c_{i-1}^{\prime}+q^{4} c_{i+1}, i \geq 0 .
$$


Proof. Set $n=N_{\pi}$. Taking the Whittaker function of $\lambda v=T v$, we have $\lambda W_{v}\left(\zeta^{i}\right)=$ $W_{T v}\left(\zeta^{i}\right), i \geq 0$. By Lemma 5.4, we obtain

$$
W_{T v}\left(\zeta^{i}\right)=W_{v^{\prime}}\left(\zeta^{i-1}\right)+\sum_{\substack{a \in \mathfrak{o}_{E} / \mathfrak{p}_{E} \\ b \in \mathfrak{p}_{F}^{-n} / \mathfrak{p}_{F}^{2-n}}} W_{v}\left(\zeta^{i} u(a, b \sqrt{\epsilon}-a \bar{a} / 2) \zeta\right) .
$$

Observe that

$$
\begin{aligned}
\zeta^{i} u(a, b \sqrt{\epsilon}-a \bar{a} / 2) \zeta & =\zeta^{i} u(a, b \sqrt{\epsilon}-a \bar{a} / 2) \zeta^{-i} \zeta^{i+1} \\
& =u\left(\varpi^{i} a, \varpi^{2 i}(b \sqrt{\epsilon}-a \bar{a} / 2)\right) \zeta^{i+1}
\end{aligned}
$$

Thus, we get

$$
\begin{aligned}
W_{v}\left(\zeta^{i} u(a, b \sqrt{\epsilon}-a \bar{a} / 2) \zeta\right) & =W_{v}\left(u\left(\varpi^{i} a, \varpi^{2 i}(b \sqrt{\epsilon}-a \bar{a} / 2)\right) \zeta^{i+1}\right) \\
& =\psi_{E}\left(\varpi^{i} a\right) W_{v}\left(\zeta^{i+1}\right)=W_{v}\left(\zeta^{i+1}\right),
\end{aligned}
$$

for $a \in \mathfrak{o}_{E}$ and $i \geq 0$ because we assume that $\psi_{E}$ has conductor $\mathfrak{o}_{E}$. Consequently, we obtain

$$
\lambda W_{v}\left(\zeta^{i}\right)=W_{v^{\prime}}\left(\zeta^{i-1}\right)+q^{4} W_{v}\left(\zeta^{i+1}\right),
$$

for $i \geq 0$. This proves the lemma.

5.2. Level lowering operator. Let $(\pi, V)$ be an irreducible generic representation of $G$ and let $n$ be an integer greater than $n_{\pi}$. Then $Z_{n-1}$ acts on $V$ trivially, and hence every vector in $V(n)$ is fixed by $Z_{n-1} K_{n}$. We define the level lowering operator $\delta: V(n) \rightarrow V(n-1)$ by

$$
\delta v=\frac{1}{\operatorname{vol}\left(K_{n-1} \cap\left(Z_{n-1} K_{n}\right)\right)} \int_{K_{n-1}} \pi(k) v d k, v \in V(n) .
$$

By the assumption $n>n_{\pi}$, we can write $\delta v$ as

$$
\delta v=\sum_{K_{n-1} / K_{n-1} \cap\left(Z_{n-1} K_{n}\right)} \pi(k) v, v \in V(n) .
$$

Lemma 5.8. Suppose that $n \geq 2$. Then a complete set of representatives for $K_{n-1} / K_{n-1} \cap\left(Z_{n-1} K_{n}\right)$ is given by

$$
t_{n-1} \hat{u}(y,-y \bar{y} / 2) \text { and } \hat{u}(a, b \sqrt{\epsilon}-a \bar{a} / 2),
$$

where $y, a \in \mathfrak{p}_{E}^{n-1} / \mathfrak{p}_{E}^{n}$ and $b \in \mathfrak{p}_{F}^{n-1} / \mathfrak{p}_{F}^{n}$.

Proof. The proof is quite similar to that of Lemma 5.2. One can easily check that

$$
K_{n-1} \cap\left(Z_{n-1} K_{n}\right)=\left(\begin{array}{ccc}
\mathfrak{o}_{E} & \mathfrak{o}_{E} & \mathfrak{p}_{E}^{1-n} \\
\mathfrak{p}_{E}^{n} & 1+\mathfrak{p}_{E}^{n-1} & \mathfrak{o}_{E} \\
\mathfrak{p}_{E}^{n} & \mathfrak{p}_{E}^{n} & \mathfrak{o}_{E}
\end{array}\right) \cap G .
$$

We set

$$
K^{\prime \prime}=\left(\begin{array}{ccc}
\mathfrak{o}_{E} & \mathfrak{o}_{E} & \mathfrak{p}_{E}^{1-n} \\
\mathfrak{p}_{E}^{n-1} & 1+\mathfrak{p}_{E}^{n-1} & \mathfrak{o}_{E} \\
\mathfrak{p}_{E}^{n} & \mathfrak{p}_{E}^{n-1} & \mathfrak{o}_{E}
\end{array}\right) \cap G
$$

Then it suffices to show the following two claims:

(i) We can take a complete set of representatives for $K_{n-1} / K^{\prime \prime}$ as the $q+1$ elements $t_{n-1}$ and $\hat{u}(0, x \sqrt{\epsilon})$, where $x \in \mathfrak{p}_{F}^{n-1} / \mathfrak{p}_{F}^{n}$.

(ii) A complete set of representatives for $K^{\prime \prime} / K_{n-1} \cap\left(Z_{n-1} K_{n}\right)$ is given by the $q^{2}$ elements $\hat{u}(y,-y \bar{y} / 2)$, where $y \in \mathfrak{p}_{E}^{n-1} / \mathfrak{p}_{E}^{n}$. 
We shall prove these claims. Clearly, the elements in (i) and (ii) are contained in pairwise disjoint cosets in $K_{n-1} / K^{\prime \prime}$ and $K^{\prime \prime} / K_{n-1} \cap\left(Z_{n-1} K_{n}\right)$ respectively.

(i) Let $k \in K_{n-1}$. If $k_{11} \in \mathfrak{p}_{E}$, then we have $t_{n-1} k \in K^{\prime \prime}$. So we get $k \in t_{n-1} K^{\prime \prime}$. Suppose that $k_{11} \in \mathfrak{o}_{E}^{\times}$. Since $k$ lies in $G$, one has $J^{t} \bar{k} J k=e$. Comparing $(3,1)$ entries, we have $k_{11} \bar{k}_{31}+k_{21} \bar{k}_{21}+k_{31} \bar{k}_{11}=0$. This implies $k_{11} \bar{k}_{31}+k_{31} \bar{k}_{11}=$ $-k_{21} \bar{k}_{21} \in \mathfrak{p}_{F}^{2 n-2}$, and hence $k_{31} \bar{k}_{11} \in \mathfrak{p}_{F}^{2 n-2} \oplus \mathfrak{p}_{F}^{n-1} \sqrt{\epsilon}$. By the assumption $k_{11} \in \mathfrak{o}_{E}^{\times}$, we have $k_{31} k_{11}^{-1}=\left(k_{11} \bar{k}_{11}\right)^{-1} k_{31} \bar{k}_{11} \in \mathfrak{p}_{F}^{2 n-2} \oplus \mathfrak{p}_{F}^{n-1} \sqrt{\epsilon}$. So there exists $x \in \mathfrak{p}_{F}^{n-1}$ such that $k_{31} k_{11}^{-1}-x \sqrt{\epsilon} \in \mathfrak{p}_{F}^{2 n-2}$. Using the assumption again, we get $k_{31}-x \sqrt{\epsilon} k_{11} \in$ $\mathfrak{p}_{E}^{2 n-2} \subset \mathfrak{p}_{E}^{n}$. This implies $k \in \hat{u}(0, x \sqrt{\epsilon}) K^{\prime \prime}$.

(ii) Let $k \in K^{\prime \prime}$. Then $\operatorname{det} k$ lies in $E^{1}$ and is equivalent to $k_{11} k_{33}$ modulo $\mathfrak{p}_{E}$. Since $k_{11}, k_{33} \in \mathfrak{o}_{E}$, we have $k_{11} \in \mathfrak{o}_{E}^{\times}$. Set $y=k_{11}^{-1} k_{21}$. Then $y$ lies in $\mathfrak{p}_{E}^{n-1}$. One can easily check that $k$ belongs to $\hat{u}(y,-y \bar{y} / 2)\left(K_{n-1} \cap\left(Z_{n-1} K_{n}\right)\right)$. This completes the proof.

By Lemma 5.8, we get an explicit formula of the level lowering operator $\delta$.

Lemma 5.9. Suppose that $n \geq 2$ and $n>n_{\pi}$. Then we have

$$
\delta v=v^{\prime}+\sum_{y \in \mathfrak{p}_{E}^{-1} / \mathfrak{o}_{E}} \pi(\zeta u(y,-y \bar{y} / 2)) v,
$$

for $v \in V(n)$.

Proof. Due to (5.7) and Lemma 5.8, we get

$$
\delta v=\sum_{\substack{a \in \mathfrak{p}_{E}^{n-1} / \mathfrak{p}_{E}^{n} \\ b \in \mathfrak{p}_{F}^{n-1} / \mathfrak{p}_{F}^{n}}} \pi(\hat{u}(a, b \sqrt{\epsilon}-a \bar{a} / 2)) v+\sum_{y \in \mathfrak{p}_{E}^{n-1} / \mathfrak{p}_{E}^{n}} \pi\left(t_{n-1} \hat{u}(y,-y \bar{y} / 2)\right) v .
$$

Here the first sum is equal to $v^{\prime}$ by (5.3). For the second sum, we use $t_{n-1}=\zeta t_{n}$ and $t_{n} \hat{u}(y,-y \bar{y} / 2) t_{n}=u\left(-\varpi^{-n} \bar{y},-\varpi^{-2 n} y \bar{y} / 2\right)$. Noting that $\pi\left(t_{n}\right) v=v$, for $v \in V(n)$, we obtain

$$
\pi\left(t_{n-1} \hat{u}(y,-y \bar{y} / 2)\right) v=\pi\left(\zeta t_{n} \hat{u}(y,-y \bar{y} / 2) t_{n}\right) v=\pi\left(\zeta u\left(-\varpi^{-n} \bar{y},-\varpi^{-2 n} y \bar{y} / 2\right)\right) v,
$$

so that

$$
\begin{aligned}
\sum_{y \in \mathfrak{p}_{E}^{n-1} / \mathfrak{p}_{E}^{n}} \pi\left(t_{n-1} \hat{u}(y,-y \bar{y} / 2)\right) v & =\sum_{y \in \mathfrak{p}_{E}^{n-1} / \mathfrak{p}_{E}^{n}} \pi\left(\zeta u\left(-\varpi^{-n} \bar{y},-\varpi^{-2 n} y \bar{y} / 2\right)\right) v \\
& =\sum_{y \in \mathfrak{p}_{E}^{-1} / \mathfrak{o}_{E}} \pi(\zeta u(y,-y \bar{y} / 2)) v .
\end{aligned}
$$

This completes the proof.

We consider the level lowering operator for $n=N_{\pi}$. Since $V\left(N_{\pi}-1\right)=\{0\}$, we have $\delta v=0$ for all $v \in V\left(N_{\pi}\right)$. For a newform $v$ in $V\left(N_{\pi}\right)$, we set $c_{i}$ and $c_{i}^{\prime}$ as in (5.5). Then we get another relation between $c_{i}$ and $c_{i}^{\prime}$.

Lemma 5.10. Suppose that $N_{\pi} \geq 2$ and $N_{\pi}>n_{\pi}$. Then we have

$$
\begin{aligned}
& c_{i}^{\prime}+q^{2} c_{i+1}=0, i \geq 0, \\
& c_{-1}^{\prime}=0 .
\end{aligned}
$$


Proof. By Lemma 5.9 and $\delta v=0$, we have

$$
0=W_{\delta v}\left(\zeta^{i}\right)=W_{v^{\prime}}\left(\zeta^{i}\right)+\sum_{y \in \mathfrak{p}_{E}^{-1} / \mathfrak{o}_{E}} W_{v}\left(\zeta^{i+1} u(y,-y \bar{y} / 2)\right)
$$

for $i \in \mathbf{Z}$. Note that $\zeta^{i+1} u(y,-y \bar{y} / 2) \zeta^{-i-1}=u\left(\varpi^{i+1} y,-\varpi^{2 i+2} y \bar{y} / 2\right)$, and hence $W_{v}\left(\zeta^{i+1} u(y,-y \bar{y} / 2)\right)=W_{v}\left(\zeta^{i+1} u(y,-y \bar{y} / 2) \zeta^{-i-1} \zeta^{i+1}\right)=\psi_{E}\left(\varpi^{i+1} y\right) W_{v}\left(\zeta^{i+1}\right)$. So we obtain

$$
0=W_{v^{\prime}}\left(\zeta^{i}\right)+W_{v}\left(\zeta^{i+1}\right) \sum_{y \in \mathfrak{p}_{E}^{-1} / \mathfrak{o}_{E}} \psi_{E}\left(\varpi^{i+1} y\right)
$$

for $i \in \mathbf{Z}$. Now the lemma follows from the assumption that $\psi_{E}$ has conductor $\mathfrak{o}_{E}$.

5.3. Zeta integrals of newforms. Let $(\pi, V)$ be an irreducible generic representation of $G$ such that $N_{\pi} \geq 2$ and $N_{\pi}>n_{\pi}$. For $v \in V\left(N_{\pi}\right)$, we set

$$
c_{i}=W_{v}\left(\zeta^{i}\right), i \in \mathbf{Z} .
$$

Then we get a recursion formula for $\left\{c_{i}\right\}_{i \in \mathbf{Z}}$.

Lemma 5.11. Suppose that $N_{\pi} \geq 2$ and $N_{\pi}>n_{\pi}$. Then we have

$$
\begin{aligned}
& \left(\lambda+q^{2}\right) c_{i}=q^{4} c_{i+1}, \quad i \geq 1, \\
& \lambda c_{0}=q^{4} c_{1} .
\end{aligned}
$$

Proof. The assertion follows from Lemmas 5.6 and 5.10

Now we get an explicit formula of zeta integrals of newforms in terms of Hecke eigenvalues.

Proposition 5.12. Suppose that an irreducible generic representation $(\pi, V)$ of $G$ satisfies $N_{\pi} \geq 2$ and $N_{\pi}>n_{\pi}$. Then we have

$$
Z\left(s, W_{v}\right)=\frac{1-q^{-2 s}}{1-\frac{\lambda+q^{2}}{q^{2}} q^{-2 s}} W_{v}(e),
$$

for $v \in V\left(N_{\pi}\right)$, where $\lambda$ is the eigenvalue of the Hecke operator $T$ on $V\left(N_{\pi}\right)$.

Proof. Since $v$ is fixed by $t(b), b \in \mathfrak{o}_{E}^{\times}$, we get $W_{v}(t(a))=W_{v}\left(\zeta^{\nu_{E}(a)}\right)$, for $a \in E^{\times}$, where $\nu_{E}$ is the valuation on $E$ normalized so that $\nu_{E}(\varpi)=1$. Proposition 2.4(i) says that $W_{v}\left(\zeta^{i}\right)=0$ for all $i<0$. So we have

$$
Z\left(s, W_{v}\right)=\int_{E^{\times}} W_{v}(t(a))|a|_{E}^{s-1} d^{\times} a=\sum_{i=0}^{\infty} W_{v}\left(\zeta^{i}\right)\left|\varpi^{i}\right|_{E}^{s-1}=\sum_{i=0}^{\infty} c_{i} q^{2 i(1-s)}
$$


By Lemma 5.11, we obtain $c_{i+1}=\left(\frac{\lambda+q^{2}}{q^{4}}\right)^{i} \frac{\lambda}{q^{4}} c_{0}$, for $i \geq 0$, and hence

$$
\begin{aligned}
Z\left(s, W_{v}\right) & =c_{0}+c_{0} \frac{\lambda}{q^{4}} \sum_{i=0}^{\infty}\left(\frac{\lambda+q^{2}}{q^{4}}\right)^{i} q^{2(i+1)(1-s)} \\
& =c_{0}+c_{0} \frac{\lambda}{q^{2}} q^{-2 s} \sum_{i=0}^{\infty}\left(\frac{\lambda+q^{2}}{q^{2}}\right)^{i} q^{-2 i s} \\
& =c_{0}+c_{0} \frac{\lambda}{q^{2}} q^{-2 s} \frac{1}{1-\frac{\lambda+q^{2}}{q^{2}} q^{-2 s}} \\
& =\frac{c_{0}\left(1-q^{-2 s}\right)}{1-\frac{\lambda+q^{2}}{q^{2}} q^{-2 s}} .
\end{aligned}
$$

This shows the proposition.

\section{Proof of Theorem 4.2}

Now we shall prove Theorem 4.2, Let $(\pi, V)$ be an irreducible generic supercuspidal representation of $G$. By Theorem 2.2 (iii), we can apply Proposition 5.12 to newforms for $\pi$. Due to Proposition 2.4(ii), we can take $v \in V\left(N_{\pi}\right)$ such that $W_{v}(e)=1$. For such $v$, we have

$$
Z\left(s, W_{v}\right)=\frac{1-q^{-2 s}}{1-\frac{\lambda+q^{2}}{q^{2}} q^{-2 s}} .
$$

It follows from the proof of Proposition 3.6 that $Z\left(s, W_{v}\right)$ is a polynomial in $q^{-2 s}$ and $q^{2 s}$. Therefore we must have $Z\left(s, W_{v}\right)=1-q^{-2 s}$ or 1 , and hence $Z\left(s, W_{v}, \Phi_{N_{\pi}}\right)=$ $Z\left(s, W_{v}\right) L_{E}(s, \mathbf{1})=1$ or $L_{E}(s, \mathbf{1})$ by Proposition 3.5 .

Suppose that $Z\left(s, W_{v}, \Phi_{N_{\pi}}\right) \neq L(s, \pi)$. Then by Proposition 3.6, we must have $Z\left(s, W_{v}, \Phi_{N_{\pi}}\right)=1$ and $L(s, \pi)=L_{E}(s, \mathbf{1})$. Thus, the functional equation

$$
\frac{Z\left(1-s, W_{v}, \hat{\Phi}_{N_{\pi}}\right)}{L(1-s, \pi)}=\varepsilon\left(s, \pi, \psi_{F}, \psi_{E}\right) \frac{Z\left(s, W_{v}, \Phi_{N_{\pi}}\right)}{L(s, \pi)}
$$

implies

$$
q^{-2 N_{\pi}(s-1 / 2)} \frac{1}{L_{E}(1-s, \mathbf{1})}=\varepsilon\left(s, \pi, \psi_{F}, \psi_{E}\right) \frac{1}{L_{E}(s, \mathbf{1})}
$$

because of Proposition 3.9. This contradicts Proposition 3.15, which states that $\varepsilon\left(s, \pi, \psi_{F}, \psi_{E}\right)$ is monomial. We therefore conclude that $Z\left(s, W_{v}, \Phi_{N_{\pi}}\right)=L(s, \pi)$. This completes the proof of Theorem 4.2

\section{ACKNOWLEDGEMEnTs}

The author would like to thank Yoshi-hiro Ishikawa for his advice and comments and Takuya Yamauchi for helpful discussions. The author also thanks the referee for careful reading of the manuscript and many suggestions on the presentation of this paper. 


\section{REFERENCES}

[1] E. M. Baruch, On the gamma factors attached to representations of $\mathrm{U}(2,1)$ over a p-adic field, Israel J. Math. 102 (1997), 317-345. MR1489111 (2000i:22019)

[2] W. Casselman, On some results of Atkin and Lehner, Math. Ann. 201 (1973), 301-314. MR.0337789(49:2558)

[3] P. Deligne, Formes modulaires et représentations de GL(2), Modular functions of one variable, II (Proc. Internat. Summer School, Univ. Antwerp, Antwerp, 1972), Springer, Berlin, 1973, pp. 55-105. Lecture Notes in Math., Vol. 349. MR.0347738 (50:240)

[4] S. Gelbart and I. Piatetski-Shapiro, Automorphic forms and L-functions for the unitary group, Lie group representations, II (College Park, Md., 1982/1983), Lecture Notes in Math., vol. 1041, Springer, Berlin, 1984, pp. 141-184. MR748507 (86f:11084)

[5] Y. Ishikawa, On standard L-function for generic cusp forms on $\mathrm{U}(2,1)$, Sūrikaisekikenkyūsho Kōkyūroku (2006), no. 1468, 46-54.

[6] H. Jacquet, I. Piatetski-Shapiro, and J. Shalika, Conducteur des représentations du groupe linéaire, Math. Ann. 256 (1981), no. 2, 199-214. MR620708(83c:22025)

[7] H. Koseki and T. Oda, Whittaker functions for the large discrete series representations of $\mathrm{SU}(2,1)$ and related zeta integrals, Publ. Res. Inst. Math. Sci. 31 (1995), no. 6, 959-999. MR $1382562(97 \mathrm{~d}: 11082)$

[8] M. Miyauchi, On local newforms for unramified U(2,1), preprint, arXiv:1105.6004, 2011.

[9] C. Mœglin, M.-F. Vignéras, and J. Waldspurger, Correspondances de Howe sur un corps padique, Lecture Notes in Mathematics, vol. 1291, Springer-Verlag, Berlin, 1987. MR1041060 (91f:11040)

[10] B. Roberts and R. Schmidt, Local newforms for GSp(4), Lecture Notes in Mathematics, vol. 1918, Springer, Berlin, 2007. MR2344630(2008g:11080)

Faculty of Liberal Arts and Sciences, Osaka Prefecture University, 1-1 Gakuen-Cho NAKAKU SAKAI, OSAKA 599-8531, JAPAN

E-mail address: michitaka.miyauchi@gmail.com 